\title{
MANIFESTACIONES ORALES EN PACIENTES CON DIABETES MELLITUS TIPO 2 ATENDIDOS EN EL HOSPITAL ALBERTO SABOGAL
}

\section{ORAL MANIFESTATIONS OF TYPE 2 DIABETES MELLITUS PATIENTS ATTENDING THE ALBERTO SABOGAL HOSPITAL}

\author{
Linares-Vega NMª, Zavaleta-Solorzano S ${ }^{1 a}$, Siapo-García Fª, Vásquez-Ramirez AJ1a, Ignacio-Cconchoy Fb, 2c
}

\section{RESUMEN}

Objetivo: Determinar las manifestaciones orales más frecuentes de la diabetes mellitus tipo 2 durante el año 2016 en el Hospital Alberto Sabogal. Materiales y Métodos: Estudio descriptivo, transversal. Se evaluó a 47 pacientes con un rango de edades de $<40$ años, 40 - 65 años y >= 65 años y en cuanto a razón femenina/masculino fue de 1/1, con diabetes mellitus tipo 2 del Hospital Sabogal, octubre de 2016. Los datos se recolectaron con una ficha que incluía variables sociodemográficas de los pacientes y evaluación de la cavidad oral. El análisis estadístico fue de distribución de frecuencias y de asociación de variables con chi $^{2}$. Resultados: El grupo etario más frecuente fue el de mayores de 65 años (48,94\%). La hipertensión arterial fue la comorbilidad más frecuente $(57,45 \%)$ y la retinopatía como complicación crónica (42,55\%). El 63,83\% usaba insulina. La manifestación oral más frecuente fue edentulismo parcial $(85,11 \%)$, seguido de caries $(82,98 \%)$, gingivitis $(78,72 \%)$, periodontitis $(57,45 \%)$, halitosis $(48,94 \%)$, lengua saburral $(40,53 \%)$, lengua fisurada (31,91\%), xerostomía (21,28\%), alteraciones del gusto $(21.28 \%)$, aftas $(14,89 \%)$, herpes $(6.38 \%)$, liquen plano $(6,38 \%)$ y abscesos $(2,13 \%)$. Ningún paciente tuvo candidiasis oral. Se obtuvo una relación significativa entre el edentulismo y el uso de insulina como tratamiento para la diabetes mellitus con un chi $^{2}=4,42$ y $p=0,03567$. Conclusiones: La manifestación oral más frecuente fue el edentulismo parcial. Se obtuvo una relación entre el edentulismo y el uso de insulina.

Palabras clave: Diabetes mellitus; manifestaciones bucales; salud bucal. (Fuente: DeCS).

\section{ABSTRACT}

Objective: To determine the most frequent oral manifestations of type 2 diabetes mellitus patients at the Sabogal Hospital during 2016. Methods: Descriptive, cross-sectional study. 47 patients were evaluated with an age range of $<40$ years, $40-$ 65 years and $>=65$ years and in the female / male ratio was 1/1, with type 2 diabetes mellitus from the Sabogal Hospital during October, 2016. Data were collected with a chart that included socio-demographic variables of the patients and oral cavity examinations. The statistical analysis was frequency distribution and association of variables with chi-square analysis. Results: The most frequent age group was, patients over 65 years of age (48.94\%). Arterial hypertension was the most frequent comorbidity with $57.45 \%$ and retinopathy as a chronic complication with $42.55 \%$. From the sample $63.83 \%$ were insulin-dependent. The most frequent oral manifestation was partial edentulism with $85.11 \%$, followed by caries with $82.98 \%, 78.72 \%$ gingivitis, $57.45 \%$ periodontitis, $48.94 \%$ halitosis, $40.53 \%$ saburral tongue, $31.91 \%$ fissured tongue, $21.28 \%$ xerostomia, $21.28 \%$ taste alterations, $14.89 \%$ had aphthas, $6.38 \%$ herpes, $6.38 \%$ lichen planus, $2.13 \%$ abscesses and no patient had oral candidiasis. A significant correlation was found between edentulism and insulin use as a treatment for diabetes mellitus with the chi-square analysis $=4.42$ and $p=0.03567$. Conclusions: The most frequent oral manifestation was partial edentulism. There was a correlation between edentulism and insulin use.

Key words: Diabetes mellitus; oral manifestations; oral health. (Source: MeSH NLM).

\footnotetext{
${ }^{1}$ Universidad San Martín de Porres.

${ }^{2}$ Hospital Nacional Alberto Sabogal Sologuren.

a Estudiantes

${ }^{b}$ Docente de Internado Hospitalario.

${ }^{c}$ Médico Internista
}

\section{Correspondencia:}

Linares Vega

Correo electrónico: nicole_linares@usmp.pe

Este es un artículo Open Access distribuido bajo la licencia Creative Commons Atribución-NoComercialCompartirlgual 4.0

\section{두(ㅇ)(잉}

Citar como: Linares-Vega NM, Zavaleta-Solorzano S, Siapo-García F, Vásquez-Ramirez AJ, Ignacio-Cconchoy F. Manifestaciones orales en pacientes con diabetes mellitus tipo 2 atendidos en el Hospital Alberto Sabogal. KIRU. 2017; 14(1):19 - 27. https://doi.org/10.24265/kiru.2017.v14n1.03 


\section{INTRODUCCIÓN}

La diabetes mellitus tipo 2 (DM) es considerada la pandemia del siglo XXI y constituye uno de los mayores problemas para los sistemas de salud. La prevalencia global de la DM está aumentando rápidamente como resultado del envejecimiento de la población, la urbanización y los cambios asociados al estilo de vida ${ }^{(1)}$. Permanece como una causa importante de morbilidad y mortalidad prematura en todo el mundo(2). En el año 2012, la Federación Internacional de Diabetes (FID) estimó que más de 371 millones de personas vivían con dicha enfermedad y que 4,8 millones de personas mueren a causa de la misma ${ }^{(3)}$. En nuestro país se estiman 1 108610 casos de diabetes mellitus tipo 2, de los cuales 8150 han fallecido a causa de dicha enfermedad $^{(4)}$. Se estima que para el año 2030 el número de personas diabéticas se incremente a 439 millones, lo que representa el $7,7 \%$ de la población adulta (de 20 a 79 años de edad) del mundo ${ }^{(5)}$.

Esta enfermedad constituye uno de los problemas metabólicos más frecuentes asociados con alteraciones del sistema estomatognático. Hoy en día, es un problema de salud creciente tanto para el mundo desarrollado como para el subdesarrollado(6). La respuesta gingival de los pacientes con diabetes no controlada ante la acumulación de placa bacteriana suele ser acentuada, de manera que la encía se torna hiperplásica y eritematosa. Entre los hallazgos periodontales que presentan los pacientes con diabetes mellitus 2 no controlada, figuran los abscesos gingivales, la proliferación de granulares subgingivales, el ensanchamiento del ligamento periodontal y la pérdida del hueso alveolar, lo cual conduce a la movilidad dentaria extrema y a la pérdida precoz de los dientes ${ }^{(7,8)}$. Por otra parte, el liquen plano bucal es un estado precanceroso vinculado frecuentemente con la diabetes mellitus, que aumenta su prevalencia cuando la glucemia supera las cifras normales. Para algunos autores, la incidencia de DM 2 en pacientes con esta afección es más alta que en la población no diabética ${ }^{(9,10)}$.

En un estudio sobre manifestaciones orales en portadores de diabetes mellitus tipo 2 de reciente diagnóstico se concluyó que el tabaquismo fue el factor de riesgo asociado más frecuente con todas las complicaciones orales seguido de la obesidad e hipertensión arterial ${ }^{(11)}$.

Otro estudio sobre manifestaciones bucales más frecuentes en pacientes diabéticos atendidos en el Instituto Autónomo Hospital Universitario de los Andes, concluyó que la gingivitis predominó en el
$97,1 \%$, seguido por caries dental en el $91,43 \%$ y periodontitis en el $85,71 \%$ de los casos. Los pacientes diabéticos tienen alta incidencia de lesiones bucales, prevaleciendo una relación altamente significativa entre la periodontitis y los pacientes diabéticos mayores de 40 años ${ }^{(12)}$.

El estudio titulado: Atención estomatológica dirigida a pacientes de nuevo ingreso en el Centro de Atención Integral del diabético, concluyó que las afecciones sistémicas fueron hipertensión arterial, vasculitis, retinopatías y en la cavidad bucal, edentulismo, periodontitis, caries crónicas y gingivitis ${ }^{(10)}$.

El estudio sobre Diabetes y enfermedad periodontal: hacia un modelo clínico bidireccional concluyó que se establece una relación desde tres hipótesis diferentes: la diabetes como factor de riesgo para la aparición y progresión de periodontitis, la infección periodontal como agravante de un difícil control metabólico y la existencia de una relación bidireccional entre ambas. Se propone un nuevo modelo clínico que explique esta relación ${ }^{(13)}$.

El objetivo general del presente estudio fue determinar las manifestaciones orales más frecuentes de la diabetes mellitus tipo 2 del Hospital Nacional Alberto Sabogal en el 2016.

Como objetivos específicos se consideró determinar las variables sociodemográficas de los pacientes con diabetes mellitus tipo 2 y establecer la relación con las manifestaciones orales en el Hospital Alberto Sabogal en el 2016.

Se justifica porque el presente estudio servirá para conocer las manifestaciones orales más frecuentes de la diabetes mellitus tipo 2 y ayudará a establecer medidas de prevención.

Se beneficiarán pacientes con diagnóstico de diabetes mellitus 2 y prediabetes así como los profesionales de la salud.

El alcance social implica que al ser la diabetes mellitus 2 un problema de salud pública contribuirá a mejorar la calidad de vida de los pacientes con afectación bucal.

\section{MATERIALES Y MÉTODO}

Se llevó a cabo un estudio observacional, descriptivo, transversal. Se evaluaron 47 pacientes con diagnóstico de diabetes mellitus tipo 2 hospitalizados en los servicios de Medicina Interna y Endocrinología del Hospital Nacional Alberto Sabogal Sologuren durante el mes de octubre de 
2016. Respecto a los criterios de selección, se incluyó a pacientes con diagnóstico de diabetes mellitus tipo 2 de ambos géneros. Se excluyó a pacientes gestantes y con trastornos neuropsiquiátricos.

Para la evaluación de cada paciente, se determinó quiénes presentaban Diabetes Mellitus tipo 2. Nos guiamos por las historias clínicas de cada uno de ellos, en las que también observamos si presentaban comorbilidades como enfermedades tardías, entre otros datos; conjuntamente se les practicó el interrogatorio y el examen bucal detallado, basado en criterios clínicos que permitieron realizar un adecuado diagnóstico, utilizando para el mismo bioseguridad en odontología como mandil, gorro, lentes, mascarilla y guante, y para la revisión intraoral: espejos bucales, exploradores y bajalenguas.

\section{FICHA DE INVESTIGACION}

Manifestaciones orales de pacientes con Diabetes Mellitus Tipo 2

Nombres y Apellidos:

H.C.:

Variables Sociodemográficas:

1. Edad: $1:<40 ; 2: 40-65 ; 3:>65$

2. Género: 1: Femenino; 2: Masculino

3. Tiempo de Enfermedad: $1:<10$ años; $2:>10$ años

4. Comorbilidad: 1. HTA; 2. Hipotiroidismo; 3. Neoplasia 4. Otros

5. Complicaciones Crónicas: 1. Retinopatía; 2. Nefropatía; 3. Neuropatía; 4. ECV; 5. IMA; 6. Pie Diabético

6. Tratamiento para DM 2: 1. Dieta; 2. Ejercicio;

3. Hipoglucemiantes Orales; 4 . Insulina

7. Procedencia:

8. Antecedentes Familiares: 1. Padre; 2: Madre; 3. Hermanos

Manifestaciones Orales:

1. Gingivitis

2. Caries

3. Periodontitis

4. Halitosis

5. Lengua Saburral

Lengua Fisurada

Xerostomía

Alteraciones del Gusto

Abscesos

10. Candidiasis

11. Aftas

12. Edentulismo

13. Herpes

14. Liquen Plano

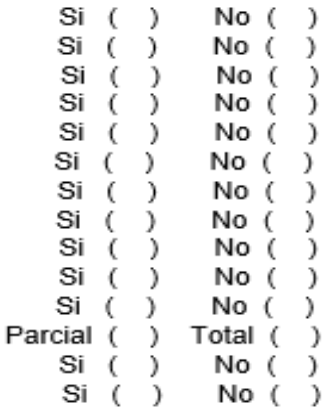

\section{RESULTADOS}

Se evaluó a 47 pacientes con diagnóstico de diabetes mellitus tipo 2, de los cuales 23 eran mujeres y 24 eran varones, hospitalizados en los servicios de Medicina Interna y Endocrinología del Hospital Nacional Alberto Sabogal Sologuren, durante el mes de octubre del año 2016. 
Tabla 1. Análisis descriptivo de las variables sociodemográficas.

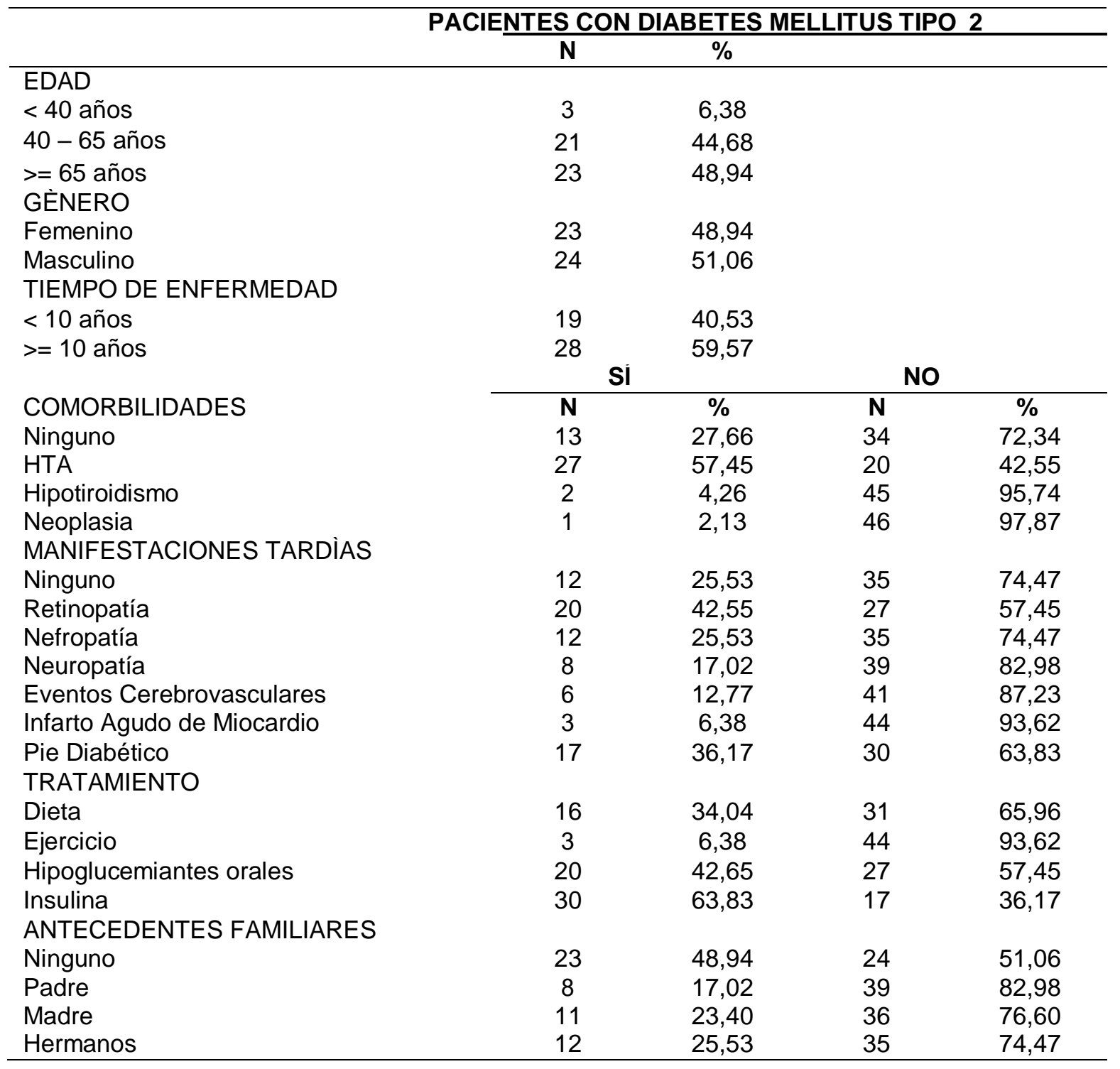

En la Tabla 1, se muestra que el grupo etario más frecuente fue el de mayores de 65 años (48,94\%) y la razón femenina/masculino fue 1/1. Los pacientes con tiempo de enfermedad mayor a 10 años fueron el $59,57 \%$.

Respecto a las comorbilidades, la hipertensión arterial fue la más frecuente con un $57,45 \%$. Las manifestaciones tardías más frecuentes fueron la retinopatías $(42,55 \%)$ y el pie diabético $(36,17 \%)$.

El $63,83 \%$ de los pacientes usaba insulina como tratamiento y la mitad tenía antecedentes de familiares diagnosticados con diabetes mellitus tipo 2. 
Tabla 2. Análisis descriptivo de las manifestaciones orales de la diabetes mellitus tipo 2.

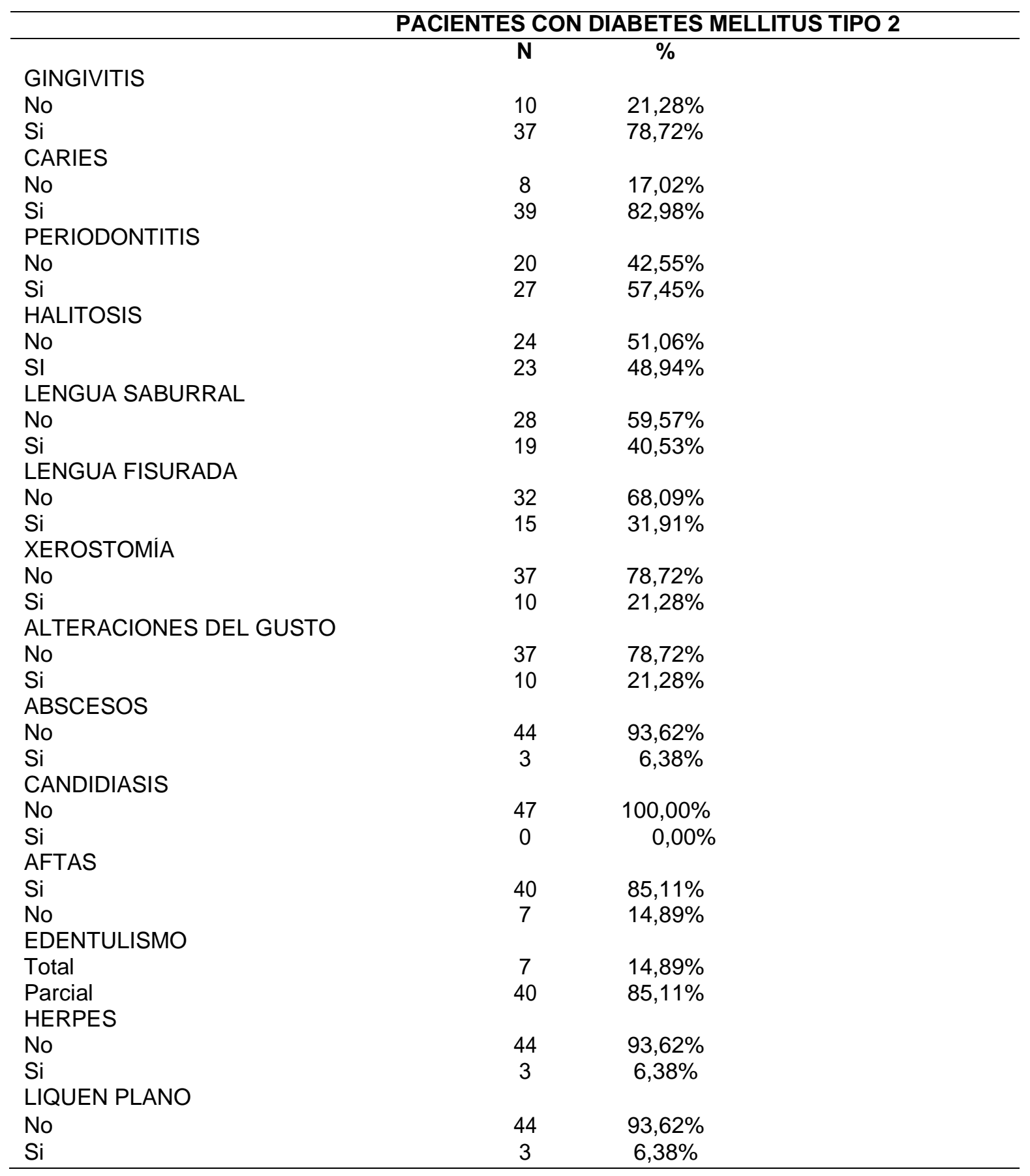

La tabla 2 muestra que el $85,11 \%$ de pacientes presentó edentulismo parcial; el $82,98 \%$ caries; el $78,72 \%$, gingivitis; el $57,45 \%$ periodontitis; el $48,94 \%$ halitosis; el 40,53\% lengua saburral; el $31.91 \%$ lengua fisurada; el 21,28\% xerostomía; el 21,28\% alteraciones del gusto; el 14,89\% aftas; el 6,38\% abscesos; el 6,38\% herpes y el $6,38 \%$ liquen plano. No hubo casos de candidiasis oral. 
Tabla 3. Análisis de frecuencia de las manifestaciones orales en relación con el uso de insulina como tratamiento de la diabetes mellitus 2

MANIFESTACIONES ORALES

\begin{tabular}{ll}
\multicolumn{1}{c}{ P } & \\
\hline EDENTULISMO PARCIAL & 0,035 \\
LENGUA SABURRAL & 0,053 \\
ALTERACIÓN DEL GUSTO & 0,077 \\
LENGUA FISURADA & 0,094 \\
HALITOSIS & 0,104 \\
HERPES & 0,256 \\
CARIES & 0,371 \\
AFTAS & 0,690 \\
ABSCESOS & 0,693 \\
XEROSTOMÍA & 0,776 \\
GINGIVITIS & 0,800 \\
PERIODONTITIS & 0,886 \\
LIQUEN PLANO & 0,916 \\
\hline
\end{tabular}

La tabla 3 se realizó con el fin de determinar si existe una asociación entre las variables tratamiento con insulina ( $\mathrm{TI})$ y las manifestaciones orales, siendo el primero el porcentaje más elevado en la tabla I. Edentulismo parcial y $\mathrm{TI}, p=0,035$; lengua saburral y $\mathrm{TI}, p=0,053$; alteración del gusto y $\mathrm{Tl}, \mathrm{p}=0,077$; lengua fisurada y $\mathrm{TI}, p=0,094$; halitosis y $\mathrm{TI}, p=$ 0,104 ; herpes y $\mathrm{Tl}, p=0,256$; caries y $\mathrm{Tl}, p=0,371$; aftas $y \mathrm{TI}, p=0,690$; abscesos $y \mathrm{TI}, p=$
0,693; xerostomía y TI, $p=0,776$; gingivitis y $\mathrm{TI}, \mathrm{p}=$ 0,800 ; periodontitis y $\mathrm{Tl}, p=0,886$, y liquen plano y $\mathrm{Tl}$, $p=0,916$.

Se obtuvo una relación significativa entre el edentulismo parcial y el uso de insulina como tratamiento para la diabetes mellitus 2 con $p=0,035$; sin embargo, no hubo relación significativa en las demás orale 
Tabla 4. Asociación de edentulismo y tratamiento con insulina.

\begin{tabular}{lccc}
\hline & \multicolumn{3}{c}{ TRATAMIENTO CON INSULINA } \\
EDENTULISMO & NO & Sí & TOTAL \\
\hline \multirow{2}{*}{ Parcial } & & & \\
& 12 & 28 & 40 \\
Total & 70,59 & 93,33 & 85,11 \\
& 5 & 2 & 7 \\
Total & 29,41 & 6,67 & 14,89 \\
& 17 & 30 & 47 \\
\hline
\end{tabular}

Pearson $\operatorname{chi}^{2}(1)=4,4288 \operatorname{Pr}=0,035$

La tabla 4 muestra que se relacionó las variables estableciendo una relación significativa entre ellas. Se obtuvo una relación significativa entre el edentulismo y el uso de insulina como tratamiento para la diabetes mellitus, con $p=0,035$.

\section{DISCUSIÓN}

En cuanto a la prevalencia de Diabetes Mellitus tipo 2 , se puede observar un incremento de acuerdo con la edad, ya que a medida que esta aumenta, disminuye la actividad física, volviéndose las personas más sedentarias y despreocupadas en cuanto a la ingesta calórica(11). El grupo etario de mayores de 65 años presenta mayor frecuencia $(48,94 \%)$ y la razón femenina/masculina es $1 / 1$. En cuanto a pacientes mayores con 10 años de tiempo de enfermedad, estos fueron el $59,57 \%$.

La diabetes mellitus 2, por sus complicaciones, disminuye la calidad de vida y va en aumento a medida que la población envejece y sufre de enfermedades cardiovasculares, siendo también la primera causa de la ceguera y amputación de miembros inferiores ${ }^{(14)}$. Se ha identificado como la comorbilidad más frecuente la hipertensión arterial $(57,45 \%)$ y como la manifestación tardía más frecuente, la retinopatía $(42,55 \%)$ seguida por pie diabético $/ 36,17 \%$ ).

Dentro del grupo de pacientes participante del estudio, el $63,83 \%$ usaba insulina como tratamiento.

El $82,98 \%$ de los pacientes participantes del estudio tenía caries dental, considerando que a medida que aumenta la edad y existe deficiencia en la higiene dental, el riesgo de presentar caries dental aumenta $^{(15)}$. El $78,72 \%$ de pacientes presentó gingivitis y la enfermedad periodontal obtuvo un porcentaje de $57,45 \%$, confirmando que existe una relación bidireccional con la diabetes Mellitus 2, siendo aquellas las enfermedades más comunes ${ }^{(16)}$.

Se observó en la evaluación que todos los pacientes habían sufrido de una a más pérdidas dentales, ya fuera por enfermedad periodontal o extracción indicada debido a un deficiente control metabólico. ${ }^{17}$ Por ello se realizó una contrastación entre la variable 'tratamiento con insulina' y las manifestaciones orales. Al aplicar el $\mathrm{chi}^{2}$ se obtuvo una relación significativa entre el edentulismo parcial y el uso de insulina como tratamiento para la diabetes mellitus, con $\mathrm{p}=0,035$.

Las enfermedades de la mucosa oral son mínimas; sin embargo, en conjunto afectan al 25 a $50 \%$ de la población. Tales afecciones son más frecuentes a medida que se envejece debido a deficiencia inmunológica, mayor frecuencia de enfermedades sistémicas y el empleo de medicamentos ${ }^{(18)}$.

No se observó candidiasis en los pacientes con diabetes mellitus tipo 2 que participaron en el estudio; sin embargo, nuestro criterio no se asemeja al de otros autores ${ }^{(19,21)}$. En dichos estudios proponen que el uso de prótesis dental es el factor causante de la candidiasis. En este estudio ninguno de los pacientes usaba prótesis parcial removible, pero 7 pacientes sí eran portadores de prótesis totales. Cabe resaltar que la higiene de estas personas, portadores de dichas prótesis, era la adecuada. 
Se concluye que el grupo etario más frecuente fue el de mayores de 65 años. La hipertensión arterial fue la comorbilidad más frecuente $(57,45 \%)$ y la retinopatía fue la manifestación tardía más frecuente $(42,55 \%)$. La insulina fue el tratamiento más frecuente $(63,83 \%)$ y la mitad de los pacientes tenían antecedentes familiares de diabetes mellitus tipo 2 . Las manifestaciones orales más frecuentes de la diabetes mellitus tipo 2 fueron: edentulismo parcial $(85,11 \%)$, caries dental $(82,98 \%)$ y gingivitis $(78,72 \%)$. Se obtuvo una relación estadísticamente significativa entre el edentulismo y el uso de insulina. No se halló candidiasis oral en los pacientes con diabetes mellitus tipo 2 examinados.

Contribuciones de autoría: FIC participó en el diseño del estudio y análisis de resultados. NLV,SZS,FSG y JVR participaron en la revisión de la literatura y recolección de datos. La redacción del artículo fue aprobado por todos los participantes.

Fuente de financiamiento: autofinanciado.

Conflictos de interés: Declaramos que no tenemos ningún interés comercial o económico con el trabajo presentado.

\section{REFERENCIAS BIBLIOGRÁFICAS}

1. Guías ALAD sobre el diagnóstico, control y tratamiento de la diabetes mellitus tipo 2 con Medicina Basada en Evidencia (Edición 2013). Rev ALAD. Consultado el 16/03/2017. 2013: 1-142 En: http://issuu.com/aladdiabetes/docs/guias_alad_2013?e=3438350/560851 4.

2. Aguirre F, Brown A, Cho N, Dahlquist G. International Federation of Diabetes. $5^{\circ}$ ed. Diabetes atlas. Consultado el 13/03/2017. 2012. En: http://www.idf.org/diabetesatlas/5e/Update2012

3. Shaw JE, Sicree RA, Zimmet PZ. Global estimates of the prevalence of diabetes for 2010 and 2030. Diabetes Rev Clin Pract. [internet] Consultado el 13/04/2017. 2010; 87: 4-14. En: https://www.ncbi.nlm.nih.gov/pubmed/19896746

4. Who.int [internet] Organización Mundial de la Salud [Consultado: 14/01/2017]. En http://www.who.int/mediacentre/factsheets/fs312/es

5. Duran $R$, Velasquez $A$, Ramirez AR, Villa BM, Mendoza ME, Tapia JA. Base de datos de defunciones 1990-2011. INEGI/Secretaría de Salud, Base de datos del SEED [internet]. Consultado el 13/01/2017. 2012. En:

http://www.dged.salud.gob.mx/contenidos/dedss/desc argas/rcs/rcs_2012.pdf
6. Consumer.es. Relación entre hábitos alimenticios y enfermedades periodontales. [Internet] 2011 [acceso 14 Ene 2017]. En: http://www.consumer.es/web/es/alimentacion/aprende r_a_comer_bien/enf ermedad/2011/10/27/204290.php

7. Diabtes.org La diabetes y los problemas de salud bucal. American Diabetes Association [internet] [Consulta: 17/01/17]. En: http://www.diabetes.org/es/vivircondiabetes/tratamiento-y-cuidado/higiene-y-saludbucal/la-diabetes-ylos-problemas-de-salud-bucal.html

8. Torres López JC, Ovalle Marroquín DF, Méndez Fernández A, Briseño M, Barrios Gallegos G, Jarquín Estrada $L$ et al. Boletín epidemiológico diabetes mellitus tipo 2. Sistema Nacional de Vigilancia Epidemiológica (EEUU). [Internet] 2013 [Consulta 17/02/2017]. 4-35. En: http://www.epidemiologia.salud.gob.mx/doctos/infoepi d/bol_diabetes/dm2_bol1_2013.pdf

9. Hechavarría Martínez $\mathrm{BO}$, Núñez Antúnez L, Fernández Toledo $\mathrm{M}$, Cobas Pérez N. Principales alteraciones bucodentales en pacientes con diabetes mellitus. MEDISAN [ Internet] 2016 [Citado 2016 Nov 27]; 20(9).

En: http://www.medisan.sld.cu/index.php/san/article/vi $\mathrm{ew} / 845$

10. Trujillo Sainz Z, Coste Reyes J, Fernández Becerra J, Henríquez Trujillo D. Atención estomatológica dirigida a pacientes de nuevo ingreso en el Centro de Atención Integral al diabético.Rev Ciencias Médicas [Internet]. 2015 Jun [Consulta: 27/11/ 2016]; 19(3): 413-422. En: http://scielo.sld.cu/scielo.php?script=sci_arttext\&pid= S1561-31942015000300005\&Ing=es.

11. Reigada A, González F, Nicolau O, Suárez Sori B. Manifestaciones orales en portadores de diabetes Mellitus tipo 2 de reciente diagnóstico. AMC [Internet] 2010 Feb [Consulta: 17/11 2016]; 14(1): En: http://scielo.sld.cu/scielo.php?script=sci_arttext\&pid= S1025-02552010000100004\&Ing=es.

12. Barrios $M$, Velazco $N$, León $M$, Pabón $A$. Manifestaciones bucales más frecuentes en pacientes diabéticos atendidos en el Instituto Autónomo Hospital Universitario de los Andes. Acta Odontológica Venezolana. [Internet] 2010 [Consulta: 14/11/2016]; 48(4): 1-8. En: http://www.actaodontologica.com/ediciones/2010/4/art 9.asp

13. Miranda M, Montoya $Y$, Saldarriaga A. Diabetes y enfermedad periodontal: hacia un modelo clínico bidireccional. Revista Nacional de Odontología [Internet] 2014 [Consulta: 01/112016]; (14), 76-87. En: http://revistas.ucc.edu.co/index.php/od/article/view/28 5

14. Ronderos M, Gomez LC, Arenas R, Duran RM, Suarez E, Franco AM. Ministerio de Salud. III Estudio Nacional de Salud Bucal-ENSAB III. II Estudio Nacional de Factores de Riesgo de Enfermedades 
15. Crónicas- ENFREC II. [Internet] 1999. [Consulta: 01/11/2016] En: http://www.visitaodontologica.co/ARCHIVOS/ARCHIV OS-

NORMAS/Salud\%20Publica_P_y_P/II_ESTUDIO_NA CIONAL_SALUD_BUCAL.pdf

16. Moya P, Caro JC, Velasquez D, Sarquis F. Caries dental y necesidad de tratamiento protésico en adultos. Comuna de Macul, 2014. Revista Dental de Chile [internet] 2015 [Consulta: 10/11/2016]; 106 (3): 25-28.

http://www.revistadentaldechile.cl/temas\%20noviembr e\%202015/pdf/6caries_dental.pdf

17. Castillo-Ghiotto G, López-Ramos R, Tineo-Tueros M, Villarreal-Neyra L, Alarcón-Palacios M. Diabetes mellitus y enfermedad periodontal: Revisión bibliográfica de la situación actual. Rev Estomatol Herediana [Internet] 2012 [Consulta: 25/11/2016]; 22(3) 183-8.

http://www.upch.edu.pe/vrinve/dugic/revistas/index.ph $\mathrm{p} / \mathrm{REH} / \mathrm{article} / \mathrm{viewFile} / 125 / 102$

18. Juárez RP, Chahín JR, Vizcaya MM, Arduña El. Salud oral en pacientes con diabetes tipo 2: caries dental, enfermedad periodontal y pérdida dentaria. Odontol. Sanmarquina [internet] 2007 [Consulta: 29/11/2016]; 10(1): 10-13. En: http://sisbib.unmsm.edu.pe/bvrevistas/odontologia/20 $07 \_n 1 / p d f / a 04 . p d f$

19. Anzola E, Galinsky D, Morales F, Salas A. Sanchez M. Organización Panamericana de la Salud. La atención de los ancianos: un desafío para los años noventa. Publicación científica No. 546 [internet] Washington 1994. [Consulta: 30/11/2016]; En: http://bases.bireme.br/cgi-

bin/wxislind.exe/iah/online/? IsisScript=iah/iah.xis\&src =google \&base $=$ PAHO\&lang $=$ \&\&nextAction $=$ Ink\&expr Search $=18407$ \&indexSearch $=$ ID

20. De Menezes Sousa MG, Lopes Costa AL, Giuseppe Roncalli A. Clinical study of the oral manifestations and related factors in type 2 diabetics patients. Braz $\mathrm{J}$ Otorhinolaryngol. 2011; 77(2): 145-52.

21. Aguado JM, Ruiz Camps I, Muñoz P, Mensa J, Almirante $B$, Vázquez $L$ et al. Recomendaciones sobre el tratamiento de la candidiasis invasiva y otras infecciones por levadura de la Sociedad Española de Enfermedades Infecciosas y Microbiología Clínica (SEIMC). Actualización 2011. Enferm Infecc Microbiol Clin. 2011; 29(5): 345-61.

22. Pappas PG, Kauffman CA, Andes D, Benjamin DK Jr, Calandra TF, Edwards JE Jr, et al. Clinical practice guidelines for the management of candidiasis: 2009 update by the Infectious Diseases Society America. Clin Infect Dis. 2009; 48(5): 503-35. 